\title{
Concurrence of Plasmodium falciparum dhfr and crt mutations in northern Ghana
}

\author{
Frank P Mockenhaupt*1, J Teun Bousema ${ }^{2}$, Teunis A Eggelte ${ }^{3}$, \\ Stephan Ehrhardt ${ }^{1,4}$, Rowland N Otchwemah ${ }^{5}$, Robert W Sauerwein ${ }^{2}$ and \\ Ulrich Bienzle ${ }^{1}$
}

\begin{abstract}
Address: ${ }^{1}$ Institute of Tropical Medicine, Charité - University Medicine Berlin, Spandauer Damm 130, 14050 Berlin, Germany, ${ }^{2}$ Dept. of Medical Microbiology, Radboud University Nijmegen Medical Centre, PO Box 9101, 6500 HB Nijmegen, The Netherlands, ${ }^{3}$ Division of Infectious Diseases, Tropical Medicine and AIDS, Academic Medical Centre, PO Box 22660, 1100 DD Amsterdam The Netherlands, ${ }^{4}$ Bernhard-NochtInstitute for Tropical Medicine, Bernhard-Nocht-Strasse 74, 20359 Hamburg, Germany and ${ }^{5}$ School of Medicine and Health Sciences, University for Development Studies, PO Box TL 1350, Tamale, Northern Region, Ghana

Email: Frank P Mockenhaupt* - frank.mockenhaupt@charite.de; J Teun Bousema - T.Bousema@ncmls.kun.nl;

Teunis A Eggelte - t.a.eggelte@amc.uva.nl; Stephan Ehrhardt - ehrhardt@bni-hamburg.de; Rowland N Otchwemah - rotchwemah@yahoo.com; Robert W Sauerwein - r.sauerwein@mmb.umcn.nl; Ulrich Bienzle - ulrich.bienzle@charite.de

* Corresponding author
\end{abstract}

Published: 15 September 2005

Malaria Journal 2005, 4:42 doi:10.1186/1475-2875-4-42

This article is available from: http://www.malariajournal.com/content/4/I/42

(c) 2005 Mockenhaupt et al; licensee BioMed Central Ltd.

This is an Open Access article distributed under the terms of the Creative Commons Attribution License (http://creativecommons.org/licenses/by/2.0), which permits unrestricted use, distribution, and reproduction in any medium, provided the original work is properly cited.
Received: 13 May 2005

Accepted: 15 September 2005

\begin{abstract}
Background: Both chloroquine (CQ) and sulfadoxine-pyrimethamine (SP) are failing drugs in much of sub-Saharan Africa. Previous findings suggest an association between resistance to CQ and to SP in vivo, in vitro, and on the molecular level.
\end{abstract}

Methods: In 126 Ghanaian children with uncomplicated malaria, associations between mutations conferring resistance in the Plasmodium falciparum dihydrofolate reductase (dhfr; SP) and chloroquine resistance transporter (crt; CQ) genes, concentrations of residual antimalarial drugs, and gametocyte carriage were examined.

Results: Mutant $\mathrm{dhfr}$ alleles and the CQ-resistance allele crt T76 were strongly associated with each other. Isolates exhibiting the $\mathrm{dhfr}$ triple mutation seven times more likely also contained crt T76 parasites as compared to isolates without the dhfr triple variant $(P=0.000 \mathrm{I})$. Moreover, both, isolates with the dhfr triple mutation (adjusted OR, $3.2(95 \% \mathrm{Cl}, \mathrm{I} .0-10.4))$ and with crt T76 (adjusted OR, I4.5 (I.4-I50.8)) were associated with an increased likelihood of pre-treatment gametocytaemia. However, crt T76 did not correlate with gametocytaemia following SP treatment and no selection of crt T76 in SP treatment failure isolates was observed.

Conclusion: These results confirm an association between $\mathrm{CQ}$ and SP resistance markers in isolates from northern Ghana. This could indicate accelerated development of resistance to SP if $\mathrm{CQ}$ resistance is already present, or vice versa. Considering the enhanced transmission potential as reflected by the increased proportion of isolates containing gametocytes when resistant parasites are present, co-resistance can be expected to spread in this area. However, the underlying mechanism leading to this constellation remains obscure. 


\section{Background}

Chloroquine (CQ) and sulfadoxine-pyrimethamine (SP) are the most frequently used antimalarial drugs in subSaharan Africa. With the spread and intensification of drug resistance of Plasmodium falciparum more effective combinatory drug regimes are now introduced in many African countries [1]. However, CQ and SP are cheap and widely available and will most likely be in use for some more years, particularly in home treatment [2-4].

In several East African countries where SP was introduced in response to intense CQ-resistance, the drug has gradually lost efficacy although the pace of this development is subject to controversy $[5,6]$. Previously, the core mutations linked with resistance to CQ and SP were found to be associated with each other in isolates from southern Ghana [7]. Although CQ and SP are structurally unrelated and the mutations conferring resistance are located on separate chromosomes [8-10], this finding suggests that parasites resistant to CQ may acquire resistance to SP more easily than sensitive ones, or vice versa. Studies in murine models [11] and in vitro [12,13] support this hypothesis. If this was true, the spread of CQ resistance could pave the way for an accelerated development of SP resistance, and thus, bear substantial importance to the health systems of affected regions. In addition, transmission potential may be increased in both SP and CQ resistant parasites $[14,15]$.

Recently, mutations in the $P$. falciparum dihydrofolate reductase (dhfr) gene in northern Ghana were observed not only to be predictive for SP treatment failure but also to be associated with increased pre-treatment gametocyte carriage [16]. Here, associations between $d h f r$ alleles, the core mutation in the $P$. falciparum chloroquine resistance transporter gene (crt T76), residual antimalarials, and gametocyte carriage in children with uncomplicated malaria were re-examined.

\section{Methods}

P. falciparum isolates were collected from children with uncomplicated malaria participating in a treatment trial in Tamale, Northern Region, Ghana, at the end of the rainy season 2002. The results of this trial and on the $d h f r$ and dihydropteroate synthetase gene (dhps) patterns are described elsewhere $[16,17]$. In the present report, data from all 126 children with complete follow-up after SP treatment for whom $d h f r$ and dhps were genotyped are analysed. The study protocol was approved by the Ethics Committee, Ministry of Health, Northern Region, and by the Health Research Unit, Ministry of Health, Accra, and parents' informed consent was obtained.

Patients were children aged 6-59 months with uncomplicated $P$. falciparum monoinfection $(\geq 2,000 \leq 200,000 / \mu \mathrm{L})$
$[18,19]$. None had severe malnutrition, a febrile disease other than malaria, "danger signs" [18], or severe malaria [20]. Treatment consisted of a single dose of SP (25/1.25 $\mathrm{mg} / \mathrm{kg}$, Fansidar, Roche, Switzerland), and success was monitored according to current guidelines $[17,19]$.

Venous blood was collected into EDTA. Asexual parasites and gametocytes were counted per $\geq 200$ and 500 white blood cells, respectively, on Giemsa-stained thick blood films, and densities were calculated based on a putative mean WBC count of $8,000 / \mu \mathrm{L}$. Gametocyte counts one week following treatment were available for 104 children. Pre-treatment levels of CQ and pyrimethamine in blood were measured by ELISA assays [21] with limits of detection of $10 \mathrm{ng} / \mathrm{mL}$ and $25 \mathrm{ng} / \mathrm{mL}$, respectively.

P. falciparum dhfr, dhps, and crt alleles were assessed by restriction fragment length polymorphisms (RFLP) of amplicons generated by nested PCR assays applying hot start procedures (HotStart Taq, Qiagen, Germany). Primers and conditions are described elsewhere $[22,23]$ as are restriction enzymes and RFLP conditions to characterize the codons $d h f r, 16,51,59,108,164$; dhps, 436, 437, 540, 581,613 ; and $c r t, 76$. Laboratory strains were used as controls for PCR and RFLP assays. Electrophoresis was perfomed on 3\% GTG agarose (FMC Bioproducts, USA) gels.

Frequencies were compared by $\chi^{2}$-test or Fisher's exact test, and continuous variables by Student's t-test, MannWhitney U-test, or Kruskal-Wallis test as applicable. Logistic regression models were used to adjust for potential confounders of the presence of resistance mutations and to identify risk factors for gametocytaemia.

\section{Results}

The median age of the 68 girls and 58 boys was 28.5 months (range, 6-59). Median axillary temperature and geometric mean parasite density were $38.8^{\circ} \mathrm{C}$ (range, $37.5-41.0$ ) and $36,358 / \mu \mathrm{L}$ (range, 2,068-175,333/ $\mu \mathrm{L}$ ), respectively. Gametocytes were found in 19\% (24/126) of the children at a geometric mean density of $50 / \mu \mathrm{L}(16-$ $1,120 / \mu \mathrm{L})$. CQ in blood was observed in $66 \%(83 / 126$; median, $32 \mathrm{ng} / \mathrm{mL}$, range, 10-718) but pyrimethamine in only two children $(78 \& 326 \mathrm{ng} / \mathrm{mL})$.

The dhfr alleles Thr-108, Val-16 and Leu-164 were not detected. Classifying mixed alleles as mutant, $d h f r$ Ile-51 occurred in 56\% (70/126), Arg-59 in 65\% (82/126), and Asn-108 in 72\% (91/126). Triple dhfr mutations (Ile-51/ Arg-59/Asn-108) were seen in 47\% (59/126), and quadruple (triple $d h f r+d h p s$ Gly-437) and quintuple (triple $d h f r+d h p s$ Gly-437/Glu-540) variants in 44\% (55/126), and $0.8 \%(1 / 126)$, respectively. Crt T76 occurred in $80 \%$ $(101 / 126)$ of the isolates. 
Table I: Associations between dhfr and crt mutations in I26 Ghanaian children with uncomplicated malaria

\begin{tabular}{|c|c|c|c|c|c|}
\hline \multirow[t]{2}{*}{ dhfr mutation } & \multirow[t]{2}{*}{ Prevalence of crt T76 mutation (\%) } & \multicolumn{4}{|c|}{ Odds ratio $(95 \% \mathrm{Cl})$ of $\mathrm{crt}$ T76 being present } \\
\hline & & Unadjusted & $P$ & Adjusted for CQ in blood* & $P$ \\
\hline \multicolumn{6}{|l|}{51} \\
\hline absent & $67.9(38 / 56)$ & $\mathrm{I}$ & & I & \\
\hline present & $90.0(63 / 70)$ & $4.3(1.5-12.5)$ & 0.002 & $5.0(1.8-13.8)$ & 0.002 \\
\hline \multicolumn{6}{|l|}{59} \\
\hline absent & $63.6(28 / 44)$ & I & & I & \\
\hline present & $89.0(73 / 82)$ & $4.6(1.7-13.0)$ & 0.0007 & $4.4(1.7-11.4)$ & 0.002 \\
\hline \multicolumn{6}{|l|}{108} \\
\hline absent & $65.7(23 / 35)$ & 1 & & 1 & \\
\hline present & $85.7(78 / 91)$ & $3.1(1.2-8.6)$ & 0.01 & $3.2(1.2-8.2)$ & 0.02 \\
\hline \multicolumn{6}{|l|}{ triple } \\
\hline absent & $68.7(46 / 67)$ & I & & I & \\
\hline present & $93.2(55 / 59)$ & $6.3(1.9-26.6)$ & 0.0006 & $7.0(2.2-22.5)$ & 0.001 \\
\hline
\end{tabular}

$\mathrm{dhfr}$, dihydrofolate reductase gene, crt, chloroquine resistance transporter gene; $95 \% \mathrm{Cl}, 95 \%$ confidence interval; CQ, chloroquine; *, Potential confounders were tested for in logistic regression models including age, sex, parasite density, dhps alleles, and axillary temperature. Adjusted odds ratios include the presence of chloroquine in blood as the only factor found to be associated (univariate odds ratio, $3.2 ; 95 \% \mathrm{Cl}, 1.2-8.6 ; P=0.0 \mathrm{I}$ ).

The concomitant occurrence of $d h f r, d h p s$, and $c r t$ alleles in identical isolates was analyzed. All $d h f r$ mutations were linked with each other. Dhfr Ile-51 was associated with Asn-108 (OR, 29.8; 95\%CI, 8.0-161, $P<0.0001)$ and Arg-59 (OR, 7.7; 95\%CI, 3.3-19,5, $P<0.0001$ ). All isolates exhibiting $d h f r$ Arg-59 additionally had Asn-108 $(P<$ $0.0001)$. Notably, in isolates with $d h f r$ variant parasites, the CQ-resistance allele crt T76 occurred significantly more frequent than in the respective wildtype isolates (Tab. 1). Separating isolates into such with e.g. $d h f r$ codon 108 pure wildtype, wildtype \& mutant, and pure mutant, the prevalence of $c r t$ T76 was $67.5 \%$ (23/35), 82.4\% (14/ $17)$, and $86.5 \%(64 / 74)$, respectively $\left(\chi_{\text {trend }}^{2}=6.1 ; P=\right.$ $0.01)$. Basically the same trend was seen for $d h f r$ codons $51(P=0.002)$ and $59(P=0.002$, data not shown $)$. Vice versa, in isolates with $c r t$ wildtype, with both $c r t$ wildtype \& T76, and crt T76 alone, the dhfr triple mutation was detected in $16 \%(4 / 25), 53 \%(9 / 17)$, and 55\% (46/84), respectively.

The prevalence of $\mathrm{crt}$ T76 increased with increasing number of any $d h f r$ mutations: it was $63 \%(20 / 32)$ in isolates with pure $d h f r$ wildtype, $74 \%(26 / 35)$ in isolates with single or double mutations, and $93 \%(55 / 59)$ in isolates exhibiting the dhfr triple mutation $\left(\chi^{2}\right.$ trend $=13.1 ; P=$ $0.0003)$. Adjusting for CQ in blood, isolates with $d h f r$ triple mutations revealed a seven-fold increased odds of concomitantly exhibiting crt T76 (Tab. 1). Dhps alleles neither correlated with $d h f r$ alleles nor with $c r t$ T76 (data not shown).

$D h f r, d h p s$, and $c r t$ alleles were examined with respect to pre-treatment gametocytaemia. As reported elsewhere
[16], children were significantly more likely to harbour gametocytes in the presence of $d h f r 51,59$, or triple mutations. This was also true for isolates exhibiting $\mathrm{crt}$ T76 (Tab. 2). Gametocytes were not observed in any $(0 / 21)$ isolate with wildtype alleles for both $d h f r$ and $c r t$, but in $16 \%$ (8/50) of isolates exhibiting either $d h f r$ or crt mutations, and in $29 \%(16 / 55)$ of isolates revealing both, $d h f r$ triple and $c r t$ T76 $\left(\chi_{\text {trend }}^{2}=8.7, P=0.003\right)$. Adjusting for additional factors influencing gametocytaemia, i.e. high parasite density arbitrarily set as $>50,000 / \mu \mathrm{L}$, the presence of CQ in blood, and axillary temperature, both the dhfr triple mutation and crt T76 were associated with an increased likelihood of gametocytaemia (Tab. 2). Dhfr alleles or $\mathrm{crt}$ T7 6 were not associated with gametocyte density (data not shown).

One week after treatment, gametocytes were observed in $63 \%(66 / 104)$ of the children. Gametocytaemia following SP treatment was associated with pre-treatment $d h f r$ mutations, e.g. dhfr Asn-108, OR 3.1; 95\%CI, 1.3-7.6. In children harbouring $\mathrm{crt}$ T76 parasites, gametocytes were only slightly more frequent $(66 \%(54 / 82))$ than in patients with $c r t$ wildtype parasites (55\% (12/22), $P=0.3$ ).

Finally, selection of $c r t$ T76 in SP treatment failures was examined. No selection was observed: In matched pairs of isolates obtained from 35 children suffering SP treatment failure, crt T76 was present in $86 \%(30 / 35)$ of pre-treatment isolates and in $80 \%(28 / 35)$ of isolates obtained at treatment failure. 
Table 2: Associations with pre-treatment gametocytemia in 126 Ghanaian children with uncomplicated malaria

\begin{tabular}{|c|c|c|c|c|c|}
\hline \multirow[t]{2}{*}{ Factor } & \multirow[t]{2}{*}{ Prevalence of gametocytemia (\%) } & \multicolumn{4}{|c|}{ Odds ratio $(95 \% \mathrm{Cl})$ of gametocytemia } \\
\hline & & Unadjusted & $P$ & Multivariate & $P$ \\
\hline \multicolumn{6}{|l|}{ CQ in blood } \\
\hline None & $27.9(12 / 43)$ & 1 & & I & \\
\hline Present & $14.5(12 / 83)$ & $0.4(0.2-1.2)$ & 0.07 & $0.3(0.1-1.0)$ & 0.04 \\
\hline Ax. temperature & n.a. & $0.3(0.1-0.6)$ & 0.0008 & $0.2(0.1-0.5)$ & 0.0009 \\
\hline \multicolumn{6}{|l|}{ Parasite density } \\
\hline$<50,000 / \mu \mathrm{L}$ & $26.3(20 / 76)$ & $\mathrm{I}$ & & I & \\
\hline$\geq 50,000 / \mu \mathrm{L}$ & $8.0(4 / 50)$ & $0.2(0.1-0.8)$ & 0.01 & $0.2(0.1-0.8)$ & 0.02 \\
\hline \multicolumn{6}{|c|}{ dhfr triple mutation } \\
\hline Absent & $10.4(7 / 67)$ & I & & I & \\
\hline Present & $28.8(17 / 59)$ & $3.5(1.2-10.2)$ & 0.009 & $3.2(1.0-10.4)$ & 0.049 \\
\hline \multicolumn{6}{|l|}{ crt 76 mutation } \\
\hline Absent & $4.0(1 / 25)$ & I & & 1 & \\
\hline Present & $22.8(23 / 101)$ & $7.1(1.0-304)$ & 0.04 & $14.5(1.4-150.8)$ & 0.02 \\
\hline
\end{tabular}

Ax., axillary; n.a., not applicable

\section{Discussion}

In this study on $P$. falciparum isolates from northern Ghana, two major findings are presented. First, SP and CQ resistance markers are strongly associated with each other, independent of residual antimalarials. Second, both are associated with an increased prevalence of gametocytes.

This study has several limitations and particularly the finding of an association between unrelated mutations needs caution in interpretation. Because polyclonal infections predominate in the area [24], the detection of a mutant allele in an isolate does not necessarily mean that all clones carry the mutation. Thus, it cannot be stated whether the linkage between the resistance markers is a true one, i.e. on the chromosomal level, or reflects cooccurrence in individual isolates. The limited number of crt wildtypes also hampered proper testing of a linkage disequilibrium. Although $d h f r$ mutations were significantly more common in the presence of $c r t \mathrm{~T} 76$ this observation needs to be construed with caution since $80 \%$ of the isolates in this study harboured the latter variant. Also, it is impossible to comment whether the presence of $\mathrm{crt}$ T76 favours the presence of $d h f r$ mutations or vice versa because this requires longitudinal observations. Likewise, the lack of temporal information impairs a clear statement on whether resistance mutations bring about increased gametocytogenesis. Due to methodology the resistance genotype of asexual parasites cannot be separated from that of gametocytes.

Despite these difficulties in drawing firm conclusions, several previous findings support the hypothesis of a linkage disequilibrium between mutations associated with SP resistance and CQ resistance. Early reports from the 1950 s and 1960s indicated that in areas or patients with established pyrimethamine-resistance in Nigeria, Burkina Faso, and Venezuela, CQ exhibited a reduced activity (reviewed in [25]). In a murine malaria model, CQ resistance could be induced in pyrimethamine-resistant parasites but not in sensitive ones [11]. In isolates from Cameroon, the in vitro activity of pyrimethamine was ten times lower in CQ resistant than in sensitive $P$. falciparum [13]. Similar but less pronounced differences have also been observed in other studies [26,27]. In southern Ghana, we previously observed that the dhfr core mutation Asn-108 was three times more likely found in isolates exhibiting crt T76 than in isolates comprising $\mathrm{crt}$ wildtype parasites [7]. The reason for this apparent association between resistance to CQ and pyrimethamine or SP is obscure since both drugs have distinct modes of action and resistance to these is determined by mutations on different chromosomes [810]. One alluringly simple explanation could be that parasites in the study area have merely become resistant to both drugs, possibly as a result of drug pressure. In this regard, previous, simultaneous or sequential treatment with CQ and SP could have selected for resistant parasites which subsequently persisted for a longer period than the drugs can be detected in blood. However, pyrimethamine was seen in only two children which indicates that it is rarely used in this community and which argues against drug-induced selection for SP resistance. In addition, the results are corrected for the presence of $\mathrm{CQ}$ in blood which can be detected for approximately one month after intake [28]. This does not exclude the possibility of selected and persisting parasites but renders it rather unlikely. Alternatively, the association between crt T76 
and $d h f r$ mutations could reflect a rapid mutator phenotype with the ability of accelerated resistance to multiple drugs. This hypothesis originates from in vitro studies observing that parasites resistant to common antimalarials acquire resistance to structurally unrelated drugs more rapidly than susceptible strains. The genetic basis of this phenomenon is unknown but suggested to involve an increased frequency of mutations per se and consequently a higher probability of modified proteins which could also include drug targets [12]. In fact, selection for highgrade pyrimethamine resistance in vitro has been shown to enhance the degree of overall genomic polymorphism [29]. In this regard, it is noteworthy that $c r t$ T76 was more frequently observed with increasing number of $d h f r$ mutations, i.e. with increasing degree of SP resistance.

In the present study, the association between the resistance markers meets with an increased presence of gametocytes in isolates comprising mutant $d h f r$ or crt alleles. Gametocytaemia seemed to reflect a rather long duration of infection as can be deduced from its low prevalence in the presence of factors suggestive for acute disease, i.e. high body temperature and parasite density, and previous CQ treatment (Tab. 2). Again, an increased frequency of resistance mutations in gametocytaemic children could result from previous drug-related selection as outlined above. However, increased gametocytaemia preceding treatment has also been observed in infections subsequently found to be CQ resistant [30-32]. Hallett et al. [15] reported that in patients with $\mathrm{crt}$ T76 parasites, gametocyte density was highly increased one week following CQ treatment. In addition, gametocytes from patients carrying crt T76 parasites produced 38 times higher oocyst burdens in the mosquito as compared to crt wildtype parasites. In Tamale, both residual CQ levels and parasites with the crt T76 mutation are abundant [33], and a clear association of crt T76 and pre-treatment gametocyte prevalence is seen. Only one crt wildtype isolate contained gametocytes impairing a sound analysis of the effect of $\mathrm{crt}$ T76 on gametocyte density. However, increased gametocyte production by $\mathrm{crt}$ T76 parasites [15] in the presence of residual CQ could partially explain the present finding. The synthesis of both, crt and $d h f r$ mutations being associated and increased gametocytaemia in their presence, gives rise to a grim scenario: Given that CQ resistant parasites have an improved transmission potential [15] the association with SP resistance would contribute to an accelerating spread of resistance to both drugs, particularly in areas where CQ resistance is frequent. In the present study, neither selection of crt T76 in SP treatment failure was observed nor a significantly elevated proportion of post-treatment gametocytaemia among children initially harbouring crt T76 parasites. Although this was not expected and would reflect an extraordinary rapid process, both observations may be influenced by the small sample size.

\section{Conclusion}

The present data provide evidence supporting a hypothesis on a connection between resistance to CQ and SP suggesting that both, CQ and SP resistance favour transmission. This needs to be verified by carefully designed longitudinal studies in regions of differing levels of drug resistance and endemicity. Per se, antimalarial treatment must be effective, and more effective than CQ and SP, not only to reduce treatment failures but also the transmission of potential co-resistance. Eventually, as this has been shown to counterbalance enhanced transmission of resistant parasites [15] the results strongly support combinatory treatment including artemisinine-derivatives.

\section{Authors' contributions}

FPM, JTB, and UB designed the study. RNO, SE and FPM were responsible for patient recruitment, clinical and parasitological examinations, and PCR assays. TAE measured drug concentrations. JTB and RWS did the gametocyte counts. FPM and JTB wrote the paper with major contributions of the other authors.

\section{Acknowledgements}

We thank the children who participated in this study and their parents as well as the members of the Northern Region Malaria Project (NORMAP). Part of this study was funded by the World Health Organization (EPH/CSR, M50-181-9).

\section{References}

I. World Health Organisation: The use of antimalarial drugs. Report of a WHO Informal Consultation 17-I7 November 2000. WHO/CDS/RBM/ 2001.33 World Health Organization: Geneva, Switzerland; 2000.

2. Foster SD: Pricing, distribution, and use of antimalarial drugs. Bull World Health Organ 1991, 69:349-363.

3. Abuaku BK, Koram KA, Binka FN: Antimalarial drug use among caregivers in Ghana. Afr Health Sci 2004, 4: I7I-I77.

4. Dzator J, Asafu-Adjaye J: A study of malaria care provider choice in Ghana. Health Policy 2004, 69:389-40I.

5. Plowe CV, Kublin JG, Dzinjalamala FK, Kamwendo DS, Mukadam RA, Chimpeni P, Molyneux ME, Taylor TE: Sustained clinical efficacy of sulfadoxine-pyrimethamine for uncomplicated falciparum malaria in Malawi after 10 years as first line treatment: five year prospective study. BMJ 2004, 28:545.

6. White N: Sulfadoxine-pyrimethamine for uncomplicated falciparum malaria: sulfadoxine-pyrimethamine is not working in Malawi. BMJ 2004, 328: 1259.

7. Mockenhaupt FP, Eggelte TA, Till H, Bienzle U: Plasmodium falciparum pfcrt and pfmdrl polymorphisms are associated with the pfdhfr NI08 pyrimethamine-resistance mutation in isolates from Ghana. Trop Med Int Health 200I, 6:749-755.

8. Wang P, Read M, Sims PF, Hyde JE: Sulfadoxine resistance in the human malaria parasite Plasmodium falciparum is determined by mutations in dihydropteroate synthetase and an additional factor associated with folate utilization. Mol Microbiol 1997, 23:979-986.

9. Peterson DS, Walliker D, Wellems TE: Evidence that a point mutation in dihydrofolate reductase-thymidylate synthase confers resistance to pyrimethamine in falciparum malaria. Proc Natl Acad Sci USA 1988, 85:9| I4-9II 8.

10. Fidock DA, Nomura T, Talley AK, Cooper RA, Dzekunov SM, Ferig MT, Ursos LM, Sidhu AB, Naude B, Deitsch KW, Su XZ, Wootton JC, 
Roper PD, Wellems TE: Mutations in the $P$. falciparum digestive vacuole transmembrane protein PfCRT and evidence for their role in chloroquine resistance. Mol Cell 2000, 6:86I-87I.

II. Powers KG, Jacobs RL, Good WC, Koontz LC: Plasmodium vinckei: production of chloroquine-resistant strain. Exp Parasitol 1969, 26:193-202.

12. Rathod PK, McErlean T, Lee PC: Variations in frequencies of drug resistance in Plasmodium falciparum. Proc Natl Acad Sci USA 1997, 94:9389-9393.

13. Ringwald P, Eboumbou ECM, Bickii J, Basco LK: In vitro activities of pyronaridine, alone and in combination with other antimalarial drugs, against Plasmodium falciparum. Antimicrob Agents Chemother 1999, 43: 1525-1527.

14. Mendez F, Munoz A, Carrasquilla G, Jurado D, Arevalo-Herrera M, Cortese JF, Plowe CV: Determinants of treatment response to sulfadoxine-pyrimethamine and subsequent transmission potential in falciparum malaria. Am J Epidemiol 2002, 156:230-238.

15. Hallett RL, Sutherland CJ, Alexander N, Ord D, Jawara M, Drakeley C], Pinder M, Walraven G, Targett GA, Alloueche A: Combination therapy counteracts the enhanced transmission of drugresistant malaria parasites to mosquitoes. Antimicrob Agents Chemother 2004, 48:3940-3943.

16. Mockenhaupt FP, Bousema JT, Eggelte TA, Schreiber J, Ehrhardt S, Wassilew N, Otchwemah RN, Sauerwein RW, Bienzle U: Plasmodium falciparum dhfr but not dhps mutations associated with sulfadoxine-pyrimethamine treatment failure and gametocyte carriage in northern Ghana. Trop Med Int Health 2005, 10:901-908.

17. Mockenhaupt FP, Ehrhardt S, Dzisi SY, Bousema JT, Wassilew N, Schreiber J, Anemana SD, Cramer JP, Otchwemah RN, Sauerwein RW, Eggelte TA, Bienzle U: A randomised, placebo-controlled, and double-blind trial on sulfadoxine-pyrimethamine alone or combined with artesunate or amodiaquine in uncomplicated malaria. Trop Med Int Health 2005, I 0:668-67I.

18. World Health Organization: Assessment of therapeutic efficacy of antimalarial drugs for uncomplicated falciparum malaria in areas with intense transmission. Document WHOIMal.96.1077 World Health Organization: Geneva, Switzerland; 1996.

19. World Health Organization: Monitoring Antimalarial Drug Resistance. Document WHO/CDS/CSR/EPH/2002.17 World Health Organization: Geneva, Switzerland; 2002.

20. World Health Organization: Severe falciparum malaria. Trans $R$ Soc Trop Med Hyg 2000, 94(suppI I):SI-90.

21. Witte AM, Klever HJ, Brabin BJ, Eggelte TA, van der Kaay HJ, Alpers MP: Field evaluation of the use of an ELISA to detect chloroquine and its metabolites in blood, urine and breast-milk. Trans R Soc Trop Med Hyg 1990, 84:52 I-525.

22. Duraisingh MT, Curtis J, Warhurst DC: Plasmodium falciparum: detection of polymorphisms in the dihydrofolate reductase and dihydropteroate synthetase genes by PCR and restriction digestion. Exp Parasitol 1998, 89:।-8.

23. Djimde A, Doumbo OK, Cortese JF, Kayentao K, Doumbo S, Diourte Y, Dicko A, Su XZ, Nomura T, Fidock DA, Wellems TE, Plowe CV, Coulibaly D: A molecular marker of chloroquine-resistant falciparum malaria. New Engl J Med 200I, 344:257-262.

24. Mockenhaupt FP, Ehrhardt S, Eggelte TA, Markert M, Anemana S, Otchwemah R, Bienzle U: Plasmodium falciparum multiplicity correlates with anaemia in symptomatic malaria. Trop Med Int Health 2003, 8:857-859.

25. World Health Organisation: Chemotherapy of Malaria. Tech Rep Ser 1961, 226:31-39.

26. Ringwald P, Bickii J, Basco LK: In vitro activity of dihydroartemisinin against clinical isolates of Plasmodium falciparum in Yaounde, Cameroon. Am J Trop Med Hyg 1999, 6 1: 187-192.

27. Pradines B, Tall A, Rogier C, Spiegel A, Mosnier J, Marrama L, Fusai T, Millet $P$, Panconi E, Trape JF, Parzy D: In vitro activities of ferrochloroquine against 55 Senegalese isolates of Plasmodium falciparum in comparison with those of standard antimalarial drugs. Trop Med Int Health 2002, 7:265-270.

28. Eggelte TA: Production of monoclonal antibodies against antimalarial drugs for use in immunoassays. In The validation of chemical and immunological tests for antimalarials in body fluids Edited by: Navaratnam V, Payne D. Centre for Drug Research International Monograph Series 3, Universiti Sains Malaysia, Penang, Malaysia; 1990:35-63.
29. Toteja R, Nair L, Bhasin VK: Genome comparison of progressively drug resistant Plasmodium falciparum lines derived from drug sensitive clone. Mem Inst Oswaldo Cruz 200I, 96:427-433.

30. Hess FI, lannuzzi A, Leafasia J, Cowdrey D, Nothdurft HD, von Sonnenburg F, Löscher T, Rieckmann KH: Risk factors of chloroquine resistance in Plasmodium falciparum malaria. Acta Trop 1996, 61:293-306.

3I. Sokhna CS, Trape JF, Robert V: Gametocytaemia in Senegalese children with uncomplicated falciparum malaria treated with chloroquine, amodiaquine or sulfadoxine + pyrimethamine. Parasite 200I, 8:243-250.

32. Sowunmi A, Fateye BA: Changes in Plasmodium falciparum gametocytaemia in children with chloroquine-sensitive asexual infections. Parasite 2003, 10:363-369.

33. Ehrhardt S, Mockenhaupt FP, Eggelte TA, Agana-Nsiire P, Stollberg K, Anemana SD, Otchwemah RN, Bienzle U: Chloroquine blood concentrations and molecular markers of chloroquine-resistant Plasmodium falciparum in febrile children in northern Ghana. Trans R Soc Trop Med Hyg 2003, 97:697-70I.

Publish with Biomed Central and every scientist can read your work free of charge

"BioMed Central will be the most significant development for disseminating the results of biomedical research in our lifetime. "

Sir Paul Nurse, Cancer Research UK

Your research papers will be:

- available free of charge to the entire biomedical community

- peer reviewed and published immediately upon acceptance

- cited in PubMed and archived on PubMed Central

- yours - you keep the copyright

Submit your manuscript here:

http://www.biomedcentral.com/info/publishing_adv.asp
BioMedcentral 